\title{
Impact dynamics of surfactant laden drops: dynamic surface tension effects
}

\author{
Mounir Aytouna $\cdot$ Denis Bartolo $\cdot$ Gerard Wegdam • \\ Daniel Bonn · Salima Rafaï
}

Received: 27 January 2009/Revised: 13 June 2009/Accepted: 15 June 2009/Published online: 7 July 2009

(C) The Author(s) 2009. This article is published with open access at Springerlink.com

\begin{abstract}
We study the impact and subsequent retraction of aqueous surfactant-laden drops upon high-speed impact on hydrophobic surfaces. Without surfactants, a rapid expansion of the drop due to the fluid inertia is followed by a rapid retraction, due to the wetting incompatibility. With surfactants, the retraction can be partly or completely inhibited. We provide quantitative measurements showing that both the expansion and the retraction dynamics depend not only on the equilibrium surface tension (ST) but also on the dynamic tension of the surfactant solutions; the latter varies significantly between different surfactants.
\end{abstract}

\section{Introduction}

Controlling drop deposition is of great importance for a wide variety of practical applications such as spray coating (Aziz and Chandra 2000), pesticide deposition on plant leaves (Wirth et al. 1991; Bergeron et al. 2000), inkjet printing (de Gans et al. 2004), bioarray design (Heller 2002) and so on. For most of these applications one seeks

M. Aytouna · G. Wegdam · D. Bonn

Van der Waals-Zeeman Institute, Amsterdam, The Netherlands

D. Bonn

Laboratoire de Physique Statistique de l'ENS,

LPS-ENS-CNRS UMR8550-P6-P7, Paris, France

D. Bartolo

Physique et mécanique des milieux hétérogènes,

PMMH-ESPCI-CNRS UMR7636-P6-P7, Paris, France

S. Rafaï $(\bowtie)$

Laboratoire de Spectrométrie Physique,

UJF-CNRS UMR5588, Grenoble, France

e-mail: rafai@spectro.ujf-grenoble.fr to optimize the coverage of the liquid and/or avoid loosing or spilling over any of the material contained in the impinging drops. Two phenomena limit the efficiency of drop deposition from sprays: splashing (Xu et al. 2005) and bouncing. Droplet rebound is the main limiting factor for deposition of small drops and/or drop impact occurring at moderate speeds (Bergeron et al. 2000). In this paper, we focus on the partial or complete rebound of drops, and ask how it can be avoided.

A typical example of a bouncing event is shown on the high speed pictures in Fig. 1a, where a millimeter-sized drop of water impacts a hydrophobic surface. In the conditions of the experiment, the early stages of the impact dynamics are dominated by a competition between inertia that drives the expansion of the drop and the surface tension cost of creating new interface. The latter also leads to the fast retraction of the drop after the maximum radius has been reached. For sufficiently large retraction velocities, the retraction ends by the formation of a liquid column that partly or completely bounces off the surface. For a given impact velocity, an obvious strategy to prevent the drop from retracting is to lower the surface tension (ST) of the liquid, thus decreasing the energy cost of creating new interface. In practice, surfactant additives are indeed included in spray formulations to enhance the deposition efficiency, for instance for increasing pesticide deposition on plant leaves (Van Valkenburg 1982). Figure 1 shows the different stages during drop impact (spreading, retraction and relaxation) for water (a) and two different surfactant solutions $(b, c)$. Figure $1 b$ demonstrates that the addition of a small amount of surfactant can completely suppress the drop rebound under otherwise identical experimental conditions. However, different surfactants turn out to behave very differently: the two drops in Fig. 1b and $\mathrm{c}$ have the same equilibrium surface tension to within 

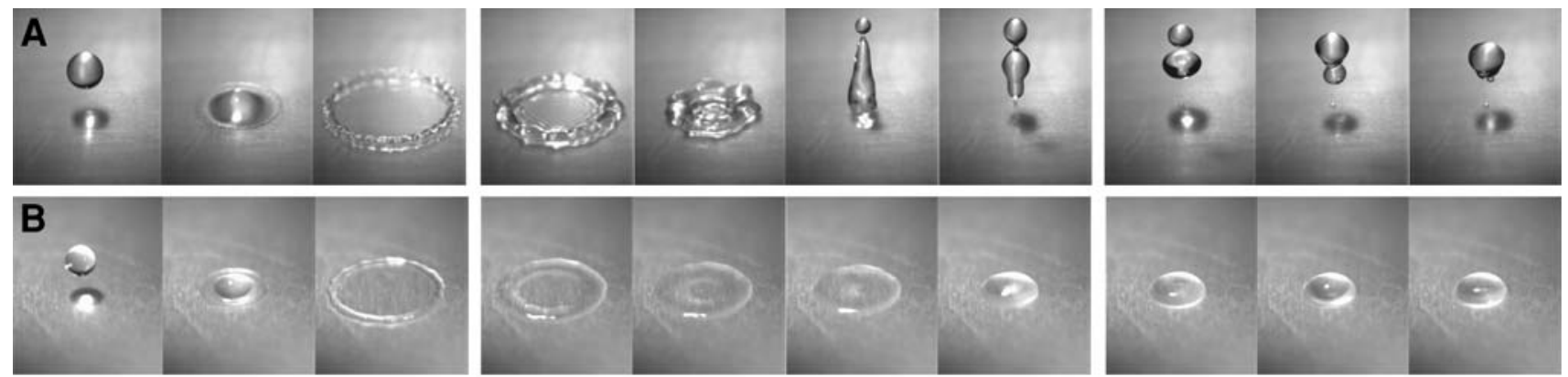

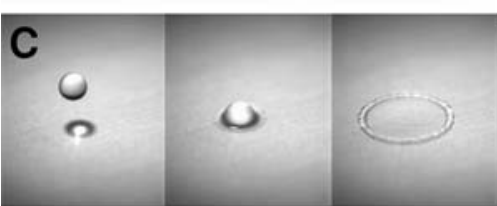

SPREADING STAGE

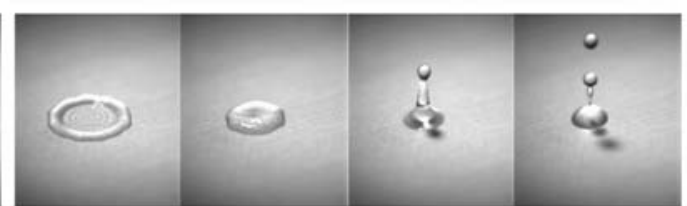

RETRACTION STAGE

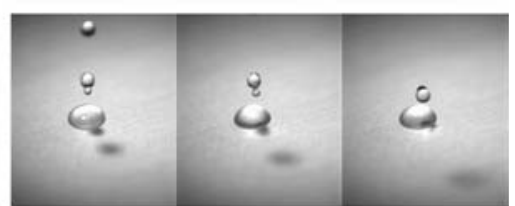

RELAXATION STAGE
Fig. 1 Impact of aqueous drops on parafilm film. Initial diameter $D_{\mathrm{I}} \sim 2 \mathrm{~mm}$, impact velocity: $V_{\mathrm{I}}=1 \mathrm{~m} / \mathrm{s}$. a Pure water, $R e=2,000$, $\mathrm{We}=28 ; \mathbf{b} 0.1 \mathrm{wt} \%$ Heliosol solution, $\gamma_{\infty}=47 \mathrm{mN} / \mathrm{m}, R e=2,000$, $\mathrm{We}=42 ;$ c $0.03 \mathrm{wt} \%$ Silwet $\mathrm{L} 77$ solution, $\gamma_{\infty}=47 \mathrm{mN} / \mathrm{m}$,

experimental accuracy. However, for the latter rebound is observed, whereas for the former it is completely suppressed. This then shows that the effect of the surfactant cannot be understood solely by considering the decrease in equilibrium surface tension caused by surfactant addition.

Our understanding of drop impact and deposition and more generally of free surface flows has substantially improved over the last 10 years. However, most works on the hydrodynamics of drop impact focus on simple fluids (Yarin 2006). So far, little attention has been paid to the effect of surfactants on the impact dynamics (MourougouCandoni et al. 1997; Zhang and Basaran 1997; Crooks et al. 2001; Marmottant et al. 2000). In their pioneering work Mourougou-Candoni et al. noted that the time taken by surfactant molecules to diffuse through to liquid to the liquid-vapor interface can be comparable to the expansion time of the impacting drop, suggesting that the interface may not be fully saturated with surfactant molecules at all times. Mourougou-Candoni et al. indeed reported a qualitative correlation between the transient or dynamic surface tension (DST) and the retraction speed of the drops of rather concentrated surfactant solutions.

In this paper, we take advantage of very recent advances in our understanding of the drop impact to shed some light on the impact dynamics of surfactant-laden drops on hydrophobic surfaces. We study the impact of aqueous solutions of surfactants that have very similar equilibrium properties (surface tension, viscosity and density) and compare results for these different surfactant solutions. Moreover, we restrict our study to low concentrations of surfactant so that bulk non-Newtonian properties can be neglected (Cooper-White et al. 2002; Rafaï et al. 2004;
$R e=2,000, \mathrm{We}=42$. We observe rebound in $\mathbf{a}$, no rebound in $\mathbf{b}$ and partial rebound in c. Typical time scales for these experiments are $2 \mathrm{~ms}$ for the spreading stage and $10-15 \mathrm{~ms}$ for the retraction stage

Bartolo et al. 1745). This allows for a clear understanding of the role of the dynamic surface tension on the dynamics of the drops. We focus our attention on two important quantities for determining deposition efficiency: the maximum radius attained by the drop and its rate of retraction. Recent experiments and theory on simple liquids (Clanet et al. 2004; Bartolo et al. 2005) provide us with a quantitative understanding of these. A systematic comparison with and without surfactants then allows us to relate the impact dynamics to the dynamic surface tension of the solutions.

\section{Experiments}

We study the impact, expansion and subsequent retraction of aqueous surfactant solution drops on parafilm, a hydrophobic surface with low-contact angle hysteresis for water. Receeding contact angles have been systematically measured for all the surfactant solutions. They are ranging from 10 to 18 for saturated solutions, the measured values are reported in the figure captions for each experiment. We have checked that the surfactants do not adsorb onto the solid. To capture the drop dynamics we use a high-speed video camera (Phantom V7 at 10,000 frames/s). The controlled release of the drops is achieved using a syringe pump to set a low drop emission rate and a syringe with a precision needle to make monodisperse drops for a given liquid. The initial diameter (before impact) $D_{\mathrm{I}}$ is systematically measured from the highspeed images: $1.5<D_{\mathrm{I}}<2 \mathrm{~mm}$. The different values of the drop radii are due to the different surface tensions of the solutions: a smaller surface tension implies that a smaller 
drop detaches from the needle. The impact speed of the drops is varied by increasing their fall height. We limit our investigation to relatively high-impact velocities. Weber (We) and Reynolds $(R e)$ numbers are both larger than $10 ; \mathrm{We} \equiv \rho$ $D_{\mathrm{I}} V_{\mathrm{I}}^{2} / \gamma$ compares inertial to capillary forces and $R e \equiv \rho D_{\mathrm{I}} V_{\mathrm{I}} / \eta$ compares inertial to viscous forces, with $V_{\mathrm{I}}$ the impact speed, $\rho$ the liquid density, $\gamma$ the liquid-vapor surface tension and $\eta$ the shear viscosity. This implies that at impact, inertial forces are at least one order of magnitude larger than both capillary and viscous forces. The drop dynamics is then governed by the competition between capillary and inertial force; viscous stresses are subdominant during the impact and expansion stages (Bartolo et al. 2005). The role of the impact speed has been studied in the range $0.7-3 \mathrm{~m} / \mathrm{s}$ which corresponds to a Weber number ranging from 10 to 700 . Within these impact conditions we are below the splashing threshold which limits the range of Weber numbers that can be explored (Xu et al. 2005); within our experimental conditions the drops thus retain their axisymmetric shape during both expansion and retraction stages.

We use aqueous solutions of different surfactants at different concentrations. The viscosity of the solutions is equal to the viscosity of water to within a few percent and independent of the shear rate, as verified by rheometry. Five types of non ionic surfactants were used: Triton $(\mathrm{X} 100, \mathrm{MW}=624 \mathrm{~g} / \mathrm{mol}), \operatorname{Agral}(90, \mathrm{MW}=603 \mathrm{~g} / \mathrm{mol})$, Silwet $(\mathrm{L} 77, \mathrm{MW}=646 \mathrm{~g} / \mathrm{mol})$; Heliosol and Li700 are naturally occurring surfactants (Terpenes and Lecitins, respectively) that have a broad molecular weight distribution. The main reason for this choice is that these surfactants offer a wide range of dynamic surface tension: Trisiloxane surfactants such as Silwet are known to be very slow (Svitova et al. 1996), whereas simple alkylethylene oxide surfactants such as Triton are known to be very fast (Fainerman et al. 1994). Heliosol is a mixture of terpenes, Agral is a nonyl phenol ethylene oxide, and consequently has the same hydrophilic moiety as Triton, and Li700 is a lecithin-based natural surfactant. All of these surfactants have been used or are still used to improve deposition efficiency in pesticide spraying. We measure the equilibrium surface tension $\gamma_{\infty}$ at different concentrations using the drop weight method. Figure 2(a) shows that the equilibrium surface tension is a decreasing function of the surfactant concentration for low concentrations, and then becomes a constant. This crossover concentration is called critical micellar concentration (CMC) and corresponds to the concentration where surfactant aggregates (micelles) start to form. We find that beyond the CMC, $\gamma_{\infty}$ is close to $35 \mathrm{mN} / \mathrm{m}$ for all the surfactants except for Silwet for which $\gamma_{\infty}=25 \mathrm{mN} / \mathrm{m}$. the CMC of Agral is around $0.1 \mathrm{wt} \%$ whereas the other surfactants have a CMC of $1 \mathrm{wt} \%$.

We also measured the dynamic surface tension of the surfactant solutions. The DST is the surface tension $\gamma(t)$ of an air/solution interface of age $t$, that had no surfactant present at time $t=0$ (Adamson 1990). We use a commercial maximum bubble pressure tensiometer (Krüss PocketDyne); typical curves for surfactant solutions decrease from high values of the ST at short times $t$ (the limit for $t=0$ should be the ST of water, $\gamma_{0}=72 \mathrm{mN} / \mathrm{m}$ ) to lower values at long time (for $t=\infty$ the ST equals the static surface tension $\left.\gamma_{\infty}\right)$. We plot the quantity $\left(\gamma(t)-\gamma_{\infty}\right) /$ $\left(\gamma_{0}-\gamma_{\infty}\right)$ in Fig. $2 b$. This quantity decreases from 1 to 0 when going from short to long times. We find that the evolution of the DST curves can be well fitted by a simple function of the form $(1+t / \tau)^{-1}$ (we use here the phenomenological model of (Hua and Rosen 1988) but set their parameter $n$ to 1 ). This allows to differentiate with a single free parameter 'fast' and 'slow' surfactants from one another, characterized, respectively by small and large characteristic times $\tau$. Clearly, these measurements indicate that the surfactants fall into two different groups: Heliosol and Triton (group I) can be considered 'fast' surfactants ( $\tau \sim 1 \mathrm{~ms}$ is an upper bound as the surfactants are so fast that we can only access the tail of the curve $\gamma(t)$ ) whereas Silwet, Agral and LI700 (group II) are significantly slower $(\tau \sim 20 \mathrm{~ms})$.

The characteristic time scale for the fast surfactants can be understood quantitatively by assuming that these present a diffusion controlled dynamics. For a diffusion controlled adsorption rate, the adsorption $\Gamma(t)$ is related to the bulk concentration $c$ via the diffusion coefficient $D$ as follows: $\Gamma(t)=2 c \sqrt{(D t / \pi)}$. Assuming that the surfactants are sufficiently dilute so that the presence of each surfactant molecule per unit of area at the interface lowers the surface tension by an amount $k_{\mathrm{B}} T$, the adsorption can be related to the surface tension. This allows to estimate the characteristic time $\tau$ for the adsorption which represents the time needed to reach the value $1 / 2$ in the curve of Fig. $2 b$, i.e. to reach half of the equilibrium adsorption $\Gamma_{\infty}$. From this simple model, it follows that $\tau(c)=(\pi / D)\left[\Gamma_{\infty} /(4 c)\right]^{2}$. Here, the only unknown is the diffusion coefficient, which can be estimated using the Stokes-Einstein equation, taking a typical value for the size of a surfactant molecule. We plot the characteristic time $\tau$ (deduced from the fits of DST measurements) as a function of concentration (Fig. 2b inset). Indeed the above equation is seen to quantitatively describe the behavior of Triton (a fast surfactant), taking $4 \mathrm{~nm}$ for the molecular size (Dong and Mao 2000).

However, a typical slow surfactant such as Agral shows a qualitatively different behavior of $\tau(c)$ (Fig. 2b, inset), with a much weaker dependence of the characteristic time on the surfactant concentration. Since this behavior is observed both below and above the CMC, it is not related to the presence of surfactant aggregates. It does, on the other hand, strongly suggest that there is another limiting factor for surfactant adsorption, such as the existence of an energy 
A

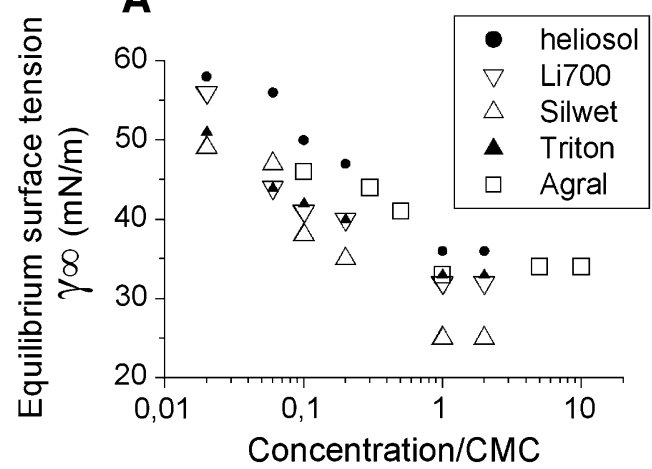

Fig. 2 a Static surface tension $\gamma_{\infty}$ of five different surfactant solutions as a function of concentration normalized by the $(\mathrm{CMC} \sim$ $0.1 \mathrm{wt} \%$ (Agral) and CMC $1 \mathrm{wt} \%$ (other surfactants)) b Relative dynamic surface tension $\left(\gamma(t)-\gamma_{\infty}\right) /\left(\gamma_{0}-\gamma_{\infty}\right)$ for 1 wt $\%$ surfactant
B

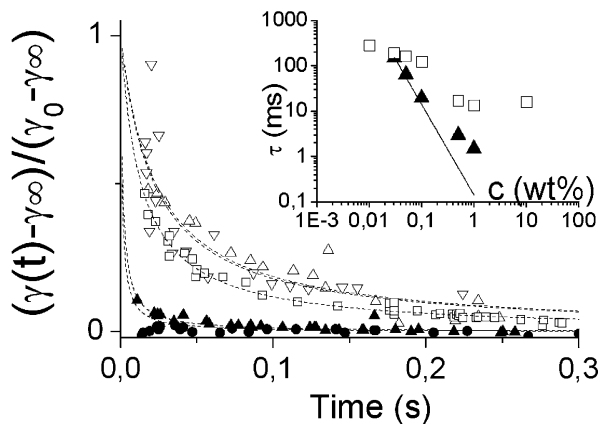

solutions. $\gamma_{0}$ is the surface tension of water. The dashed lines are best fits to an hyperbolic function as explained in the text. Inset Characteristic time scale $\tau$ as a function of concentration of Agral and Triton, the solid line is a quadratic fit
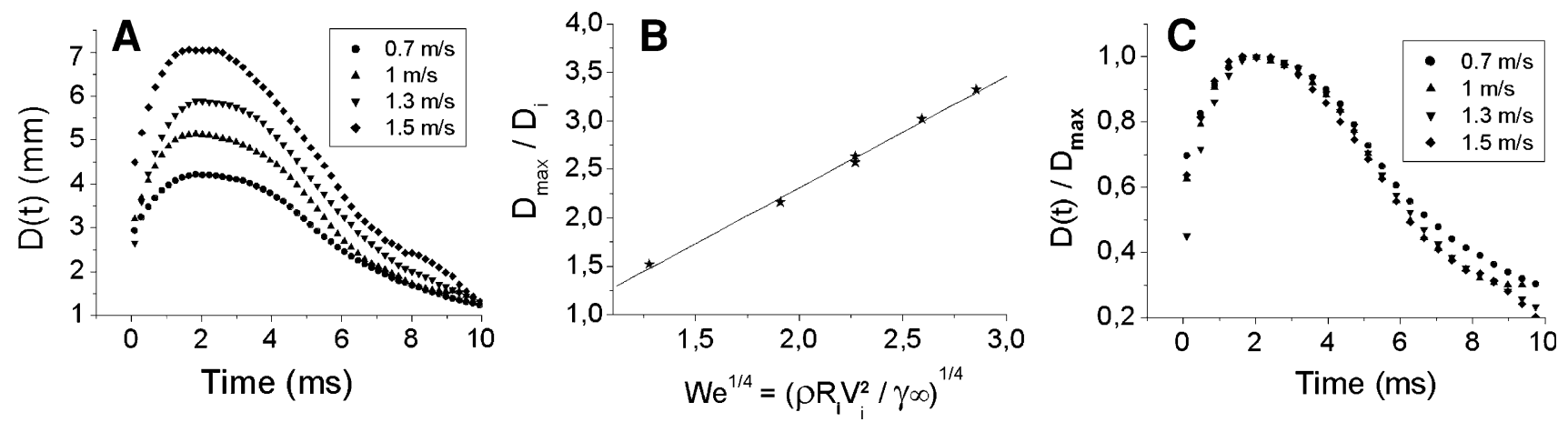

Fig. 3 Impact of a water drop of initial diameter $D_{\mathrm{I}} \sim 2 \mathrm{~mm}$ on parafilm. a Liquid-substrate contact diameter of the drop as a function of time for four different impact velocities. b Maximal spreading diameter rescaled by the initial diameter as a function of $\mathrm{We}^{1 / 4}$.

barrier for the transfer of the individual surfactant molecules from the bulk onto the surface (Chang and Franses 1995).

\section{Results and discussion}

For drop impact and retraction, despite the complex multiscale interplay between viscous, capillary and inertial stresses two simple semi-quantitative pictures have been recently proposed to account for the observed maximal expansion and for the retraction velocities of impacting drops. We first recall these recent results that we will use as guidelines for our study of the effects of the dynamic surface tension effect.

First, Clanet et al. (2004) showed that the maximal spreading diameter $D_{\max }$ should scale as $D_{\mathrm{I}} \mathrm{We}{ }^{1 / 4}$ for lowviscous liquids; this scaling holds for almost four decades in We with a prefactor close to unity. Our data for different impact speeds of water drops (see Fig. 3) confirm this c Contact diameter of the drop rescaled by the maximum diameter as a function of time for four different impact velocities. $R e$ varies from 1,400 to 3,000

result in our experimental setup: we find the same scaling behavior, Fig. 3b, with a numerical factor of 1.1 .

Second, Bartolo et al. (2005) measured the retraction rate of drops upon drop impact (defined as the retraction velocity divided by the maximum diameter $V_{\text {ret }} / D_{\max }$ ). They demonstrated that for high-speed (inertial) impacts the retraction rate is independent of the impact speed. This can be understood by writing down a force balance for the motion of the contact line. The temporal evolution of water drop contact diameters (diameter of the solid area in contact with the drop) is plotted for different impact velocities in Fig. 3a as a test. In Fig. 3c we show that indeed, independently of the impact speed, the rescaled $D / D_{\max }$ curves collapse onto a single curve.

\subsection{Results: concentrated surfactant solutions above the CMC}

Figure 4 shows the contact diameter $D(t)$ rescaled by the initial drop diameter $D_{\mathrm{I}}$ as a function of time for different 


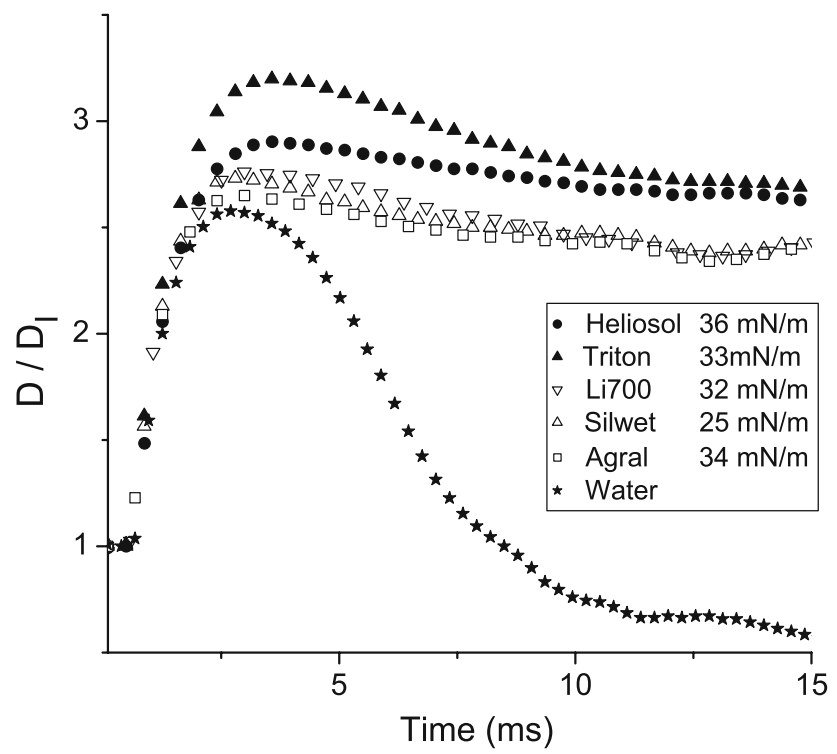

Fig. 4 Impact on parafilm of surfactant drops above the CMC compared to water impact: rescaled contact diameter by the initial diameter $D_{\mathrm{I}} \cdot R e=2,000$ and $W e$ varies from 28 (water) to 79 (Silwet)

rather concentrated surfactant solutions ( $1 \mathrm{wt} \%$, which exceeds the CMC for all these surfactants by at least a factor of two). It is observed that $D_{\max }$ increases and $V_{\text {ret }}$ is substantially reduced by all the surfactants. In addition, it is observed that although these solutions have very similar equilibrium surface tensions, their $D(t)$ curves are markedly different.

\subsubsection{Maximum diameter}

The most striking difference between the five curves is the maximum diameter attained by the drops. It appears that the large variations of $D_{\max }$ observed are not correlated with the slight variations of $\gamma_{\infty}$ between the different solutions. To identify the origin of the scatter in the data, in Fig. 5a we plot the maximum elongation $D_{\max } / D_{\mathrm{I}}$ as a function of $\mathrm{We}^{1 / 4}$. From this, we infer that only the drops containing group I (fast) surfactants behave in a similar way as simple liquids: the data fall on a straight line of slope $\sim 1$. Note here that our aim is not to demonstrate the scaling [studied in detail in (Clanet et al. 2004)] but to study the different behavior of surfactant solutions compared to simple liquids. Since We scales as $1 / \gamma$, the deviation of the group II data from the power law behavior can be directly translated into a difference in the DST during the expansion stage. The variations of the corresponding effective surface tension, $\gamma_{\text {eff }}$, with $\mathrm{We}$ are shown in Fig. 5b. For the group II surfactants, $\gamma_{\text {eff }}$ is twice larger than $\gamma_{\infty}$ and does not vary significantly with the (impact) Weber number. The conclusion is that group II surfactants hardly reduce the surface tension with respect to that of pure water: for Agral and LI700 $\gamma_{\text {eff }}$ has a value very close to the equilibrium surface tension of water. The weak variations of $\gamma_{\text {eff }}$ with $W e$ can be understood by noting that the time taken by the liquid to reach its maximal extension is $\sim 2 \mathrm{~ms}$ and hardly changes with the impact velocity (Fig. 6b). This suggests that the time taken by 'slow' surfactant molecules to reach the expanding interface is much larger than $2 \mathrm{~ms}$, and that for the 'fast' surfactants significantly smaller. This agrees with the DST measurements from which we inferred a characteristic time of $\sim 20 \mathrm{~ms}$ for slow, and $\sim 1 \mathrm{~ms}$ for 'fast' surfactants. Therefore, although the free surface dynamics is different from that of the drop impact, the maximum bubble pressure DST measurements appear to provide a correct estimate for the surface tension relaxation time of $\gamma(t)$ for the drops.

\subsubsection{Retraction rate}

Contrary to what happens for the spreading stage, the drop retraction stage in the presence of surfactants is very similar to that observed for simple liquids. Once the maximum diameter is reached, the $D(t) / D_{\max }$ curves almost coincide for surfactant solutions having very similar equilibrium surface tensions $\gamma_{\infty}$, Figure 6(a). Even for slow surfactants such as Agral, the retraction rate is observed to be
Fig. 5 a Maximal diameter of the spreading drop normalized by the initial drop radius as a function of the Weber number (log-log scale). The solid line indicates the slope $\mathrm{We}^{1 / 4}$ with a prefactor of 1.1. $\mathbf{b}$ Effective surface tension as a function of the impact Weber number. $\gamma_{\text {eff }}$ is defined as the deviation to solid line fit in (a)
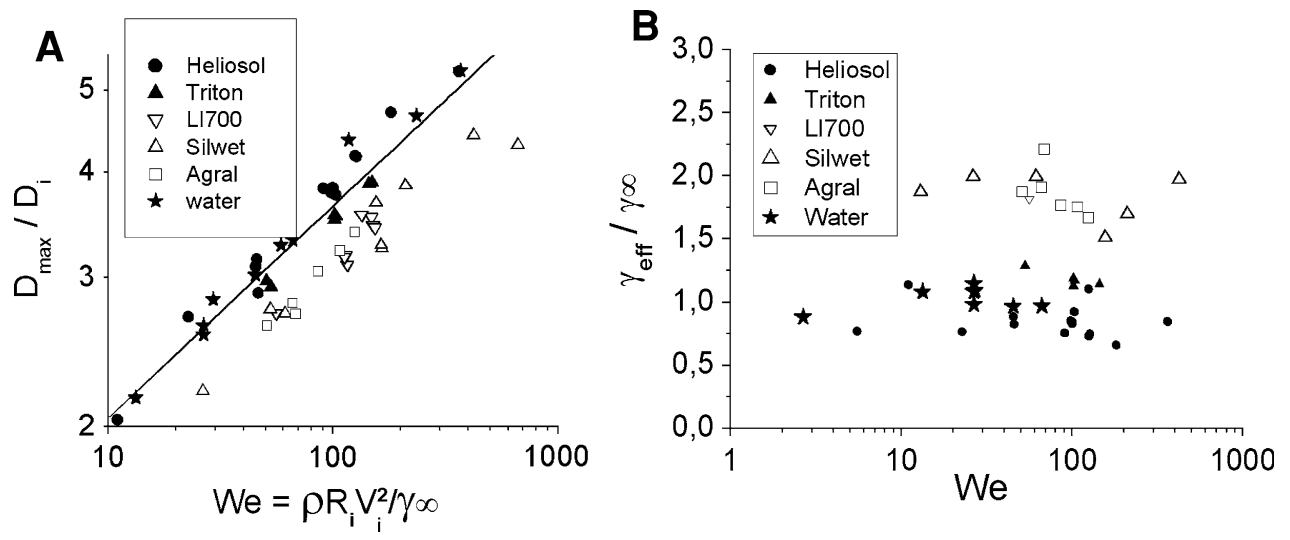


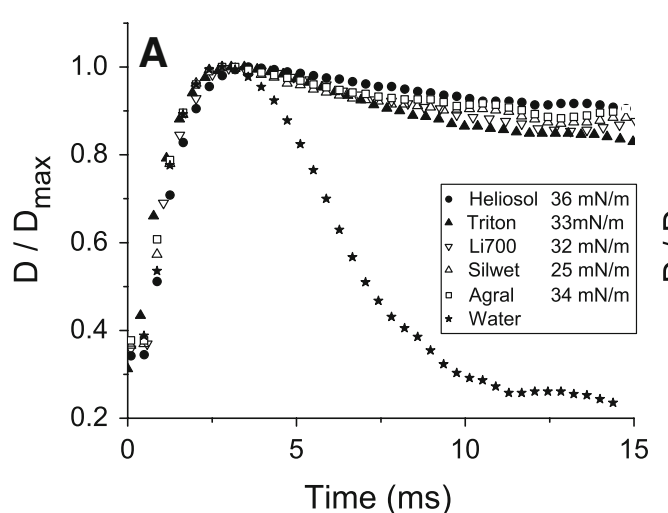

Fig. 6 Impact on parafilm of surfactant drops above the CMC: rescaled contact diameter by the maximal diameter. a Different surfactant solutions impacting at $1.1 \mathrm{~m} / \mathrm{s}$. $R e=2,200$ and We varies

independent of the impact velocity, as previously observed for pure liquids. These features are necessarily associated with a constant surface tension, and the conclusion must therefore be that the surface tension of surfactant-laden drops reaches its equilibrium value at the onset of the drop retraction.

This can be understood as follows. Before the liquid hits the solid surface, we can reasonably assume that $\gamma=\gamma_{\infty}$. As the drop expands, the rate of growth of the free liquid surface decreases from $\sim V_{\mathrm{I}} / R_{\mathrm{I}}>10^{3} / \mathrm{s}$ at short times to zero at late times. The characteristic time for the arrival of the surfactant molecules was found to be larger than $10^{-3} \mathrm{~s}$. Subsequently, $\gamma$ increases as free surface is created faster than the diffusion time of the surfactant molecules. At later times, the rate of creation of the interface becomes sufficiently small to allow the surface tension to decrease again to a value very close to $\gamma_{\infty}$ when the drop has reached its maximal diameter. This is the only possible explanation that we have found for the results of Fig. $6 a$ and $b$ that clearly indicate that the retraction stage is characterized by a constant surface tension.

\subsection{Results: dilute surfactant solutions below the CMC}

We now investigate the impact and retraction dynamics of rather dilute surfactant solutions, below the CMC. Figure 7 compares the time evolution of the (rescaled) drop radius for pure water to Triton (group I) and Agral (group II) containing drops at concentrations $c=0.1$ and $0.5 \mathrm{CMC}$, respectively. The amounts of Triton and Agral have been chosen to obtain identical equilibrium surface tensions of $40 \mathrm{mN} / \mathrm{m}$ for the two surfactant solutions. Contrary to what was observed for the more concentrated solutions, here the presence of the surfactants does not change the expansion stage of the drop at all. We find that the $D(t)$ curves for the

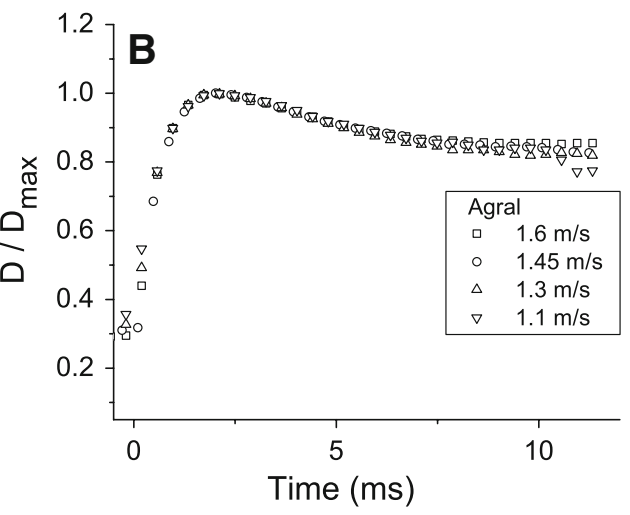

from 34 (water) to 95 (Silwet). b Agral drops at different impact velocities. $R e$ ranges from 2,200 to 3,500 and We ranges from 70 to 150

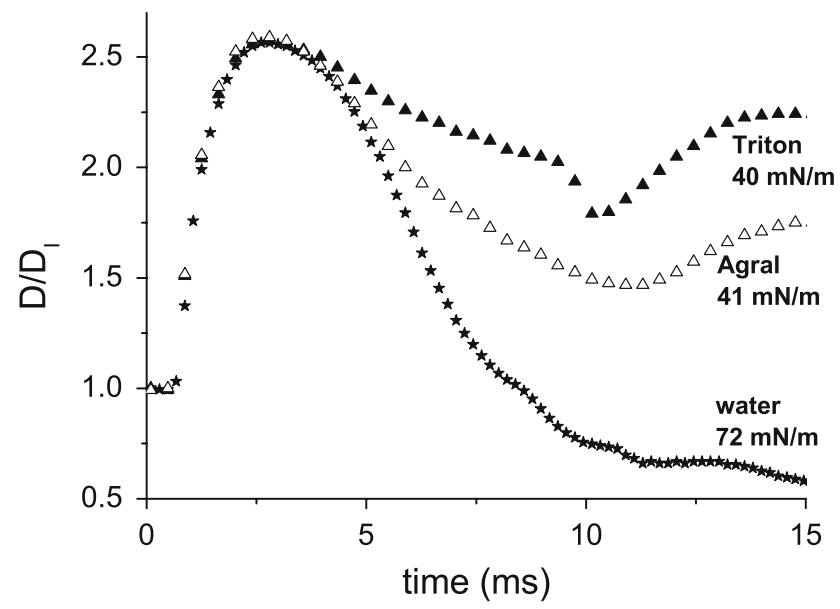

Fig. 7 Diameter of the drops (rescaled with the initial diameter $D_{\mathrm{I}}$ ) versus time for two different surfactant solutions solutions compared to that for water. These two surfactant solutions have similar equilibrium properties: liquid-vapour surface tensions (Agral: $41 \mathrm{mN} / \mathrm{m}$ and Triton: $40 \mathrm{mN} / \mathrm{m}$ ) and equilibrium receding contact angles (Agral: $14^{\circ}$ and Triton: $13^{\circ}$ ). (We display here one third of the data actually taken for the sake of clarity)

surfactant-containing and pure water drops are identical. In other words, for the dilute suspensions the effective surface tension defined in the previous section is $\gamma_{\text {eff }} \sim \gamma_{\text {water }}$ even for the group I surfactants. A second important difference with the dynamics of concentrated solutions is found for the drop retraction. Here, the retraction rate depends strongly on the type of surfactant despite the fact that their equilibrium ST are identical and that the two liquids have the same equilibrium receding contact angle on the parafilm surface $\left(14^{\circ}\right)$. The $D(t)$ curves for surfactant drops deviate from that of pure water and consequently the surfactants have not reached their equilibrium interface concentration at the onset of retraction, although the expansion time is identical to that for concentrated drops (see Figs. 4, 7). 

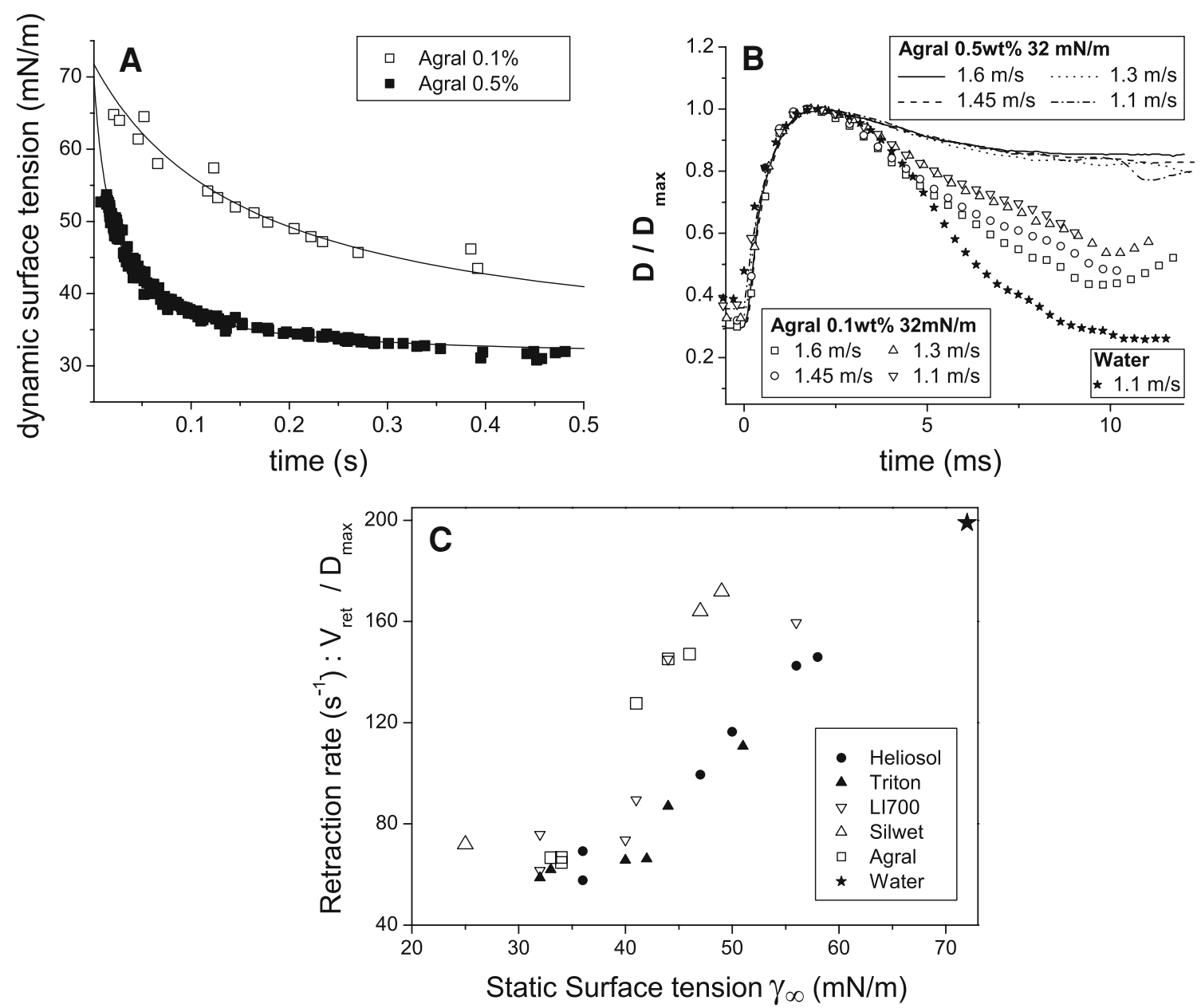

Fig. 8 a Dynamic surface tension of two Agral solutions with concentrations $1 \times \mathrm{CMC}$ (square) and $5 \times \mathrm{CMC}$ (filled square). b Rescaled diameter $\left(D / D_{\max }\right)$ of the same Agral solutions drops for different impact velocities. Again, the equilibrium properties are the

To further investigate the effect of concentration on the retraction dynamics, we also compare two solutions of Agral of different concentrations: a dilute one at $c=0.1 \mathrm{wt} \%$ and a concentrated one at $c=0.5 \mathrm{wt} \%$ $(\mathrm{CMC}=0.1 \mathrm{wt} \%)$, which have identical equilibrium surface tension $(34 \mathrm{mN} / \mathrm{m})$ but different relaxation times in the DST measurement (Fig. 8a). Figure 8(b) shows the results of impact experiments done with Agral drops at different impact velocities for the two different concentrations. We observe that the spreading dynamics is very similar but the retraction rate strongly varies between the two concentrations. As shown above, the retraction rate is not changed by changing the impact velocity for concentrated solutions. Beyond the CMC, the surfactant-laden drops behave just like a pure liquid with $\gamma=\gamma_{\infty}$ as far as the retraction is concerned. On the other hand, for the 'dilute' solution the retraction rate strongly increases (in absolute value) with increasing impact velocity. The latter observation can be understood using the following simple picture. In the very same $\left(\gamma_{\infty}=34 \mathrm{mN} / \mathrm{m}\right.$ and receding contact angle is about $\left.14^{\circ}\right)$. c Retraction rate $\dot{\epsilon}$ as a function of static surface tension for the different surfactant solutions and for water

dilute limit, the amount of surfactant adsorbed on the interface $\Gamma$ directly gives the surface tension decrease as $\gamma_{\text {water }}-\gamma=\mathcal{N}_{\mathrm{A}} k_{\mathrm{B}} T \Gamma$, with $\mathcal{N}_{\mathrm{A}}$ the Avogadro number, and $k_{\mathrm{B}} T$ the thermal energy. The adsorption in the pancakeshaped drop at the maximal extension time $t_{\max }$ can be approximated by: $\Gamma\left(t=t_{\max }\right)=\Gamma(t=0)\left(2 D_{\mathrm{I}} / D_{\max }\right)^{2}$, and $D_{\max } / D_{\mathrm{I}} \sim \mathrm{We}^{1 / 4}$. At the onset of retraction the actual surface tension driving the retraction increases with the stretching of the drop and so with the impact Weber number as:

$\gamma\left(t_{\text {max }}\right)=\gamma_{\text {water }}-\frac{\left(\gamma_{\text {water }}-\gamma_{\infty}\right)}{\mathrm{We}^{1 / 2}}$

This equation is valid if one assumes that the expansion of the interface is so fast that new surfactant molecules can not adsorb. Similarly to the inertial dewetting of thin films, the retraction rate scales as $\gamma^{1 / 2}$ for inertio-capillary drop retraction. The above equation thus correctly predicts the qualitative trend observed in Fig. 8: a decrease of the 
retraction rate with increasing impact velocity. Such an effect is also expected to affect the dynamics of the receding contact angle during retraction as one can note that the equilibrium values of the receding contact angle cannot explain the different behaviors (Figs. 7, 8a, b). The retraction dynamics is strongly affected by the adsorption kinetics of the surfactants molecules. This can be transposed in terms of effective surface tension and effective contact angles.

To complete our study, we also measure the retraction rate $\dot{\epsilon}$ for different surfactants at various concentrations. Figure 8(c) depicts the retraction rate as a function of the ST for different solutions of surfactants. The data show significant differences between the different surfactants: for a given equilibrium surface tension, depending on the type of surfactant the retraction rates can be different by up to a factor of two. The data suggest again that we can define two groups of surfactants: Heliosol and Triton on the one hand (group I) and LI700, Silwet and Agral on the other (group II). The first group presents a significantly slower retraction than the second group. This suggests that the effective ST driving the retraction is lower in the case of group I than in the case of group II. This observation is qualitatively correlated with the measurements of the dynamic surface tension where group I (resp. group II) corresponds to fast (resp. slow) surfactants. However, we recall that the consequences of surfactant addition for the retraction stage is mainly due to a concentration effect; indeed, for the high-concentration samples (lowest equilibrium ST), the different surfactant solutions behave similarly as far as the retraction rate is concerned. For intermediate values of the ST (from 40 to $60 \mathrm{mN} / \mathrm{m}$ in Fig. 8c, i.e. for concentrations lower than the CMC), the two different cases depicted in Fig. 8c are observed. This is again a signature of strong difference in the dynamic surface tension of the surfactants. As we explained above, the retraction stage in the case of low-concentration samples is governed by the ability of surfactant molecules to diffuse and adsorb to the air-water interface quickly enough to significantly reduce the ST. Figure 8c tells us that surfactants belonging to group I are therefore more efficient for preventing drop rebound as they lead to a much smaller retraction rate than those of group II.

\section{Conclusion}

We studied the effect of the dynamic surface tension of surfactant solutions on inertially dominated impact of aqueous surfactant solutions on hydrophobic surfaces. We find that when surfactants are added there are significant differences in the expansion and retraction behavior compared to pure liquids. This is concluded from a detailed comparison between the results for chemically different surfactant molecules and comparison with previous results on impact without surfactants. Our study allows to distinguish two families of surfactants: 'fast' surfactants that are able to reach their equilibrium surface tension in a short time $(\sim 1 \mathrm{~ms})$ compared to 'slow' surfactants $(\sim 20 \mathrm{~ms})$. In addition to the surfactant concentration, the rapidity of adsorption of the surfactants influences both the maximum spreading diameter and the retraction rate.

Open Access This article is distributed under the terms of the Creative Commons Attribution Noncommercial License which permits any noncommercial use, distribution, and reproduction in any medium, provided the original author(s) and source are credited.

\section{References}

Adamson AW (1990) Physical chemistry of surfaces. Wiley, New York

Aziz SD, Chandra S (2000) Impact, recoil and splashing of molten metal droplets. Int J Heat Mass Trans 43(16):2841

Bartolo D, Josserand C, Bonn D (2005) Retraction dynamics of aqueous drops upon impact on non-wetting surfaces. J Fluid Mechanics 545:329

Bartolo D, Boudaoud A, Narcy G, Bonn D (2007) Dynamics of nonNewtonian droplets. Phys Rev Lett 99:174502

Bergeron V, Bonn D, Martin JY, Vovelle L (2000) Controlling droplet deposition with polymer additives. Nature 405:772

Chang CH, Franses EI (1995) Adsorption dynamics of surfactants at the air/water interface: a critical review of mathematical models, data, and mechanisms. Colloids Surf A 100:1

Clanet C, Beguin C, Richard D, Quere D (2004) Maximal deformation of an impacting drop. J Fluid Mechanics 517:199

Cooper-White JJ, Crooks RC, Boger DV (2002) A drop impact study of worm-like viscoelastic surfactant solutions. Colloids Surf A: Physicochem Eng Aspects 210(1):105

Crooks R, Cooper-White J, Boger DV (2001) The role ofdynamic surface tension and elasticity on the dynamics of drop impact. Chem Eng Sci 56:5575

de Gans BJ, Duineveld PC, Schubert US (2004) Inkjet printing of polymers: state of the art and future developments. Adv Mater 16(3):203

Dong J, Mao G (2000) Direct study of C12E5 aggregation on mica by atomic force microscopy imaging and force measurements. Langmuir 16:6641

Fainerman VB, Miller R, Joos P (1994) The measurement of dynamic surface-tension by the maximum bubble pressure method. Colloid Polym Sci 272(6):731

Heller MJ (2002) DNA microarray technology: devices, systems, and applications. Annu Rev Biomedical Eng 4:129

Hua XY, Rosen MJ (1988) Dynamic surface-tension of aqueous surfactant solutions. 1. Basic parameters. J Colloid Interface Sci 124(2): 652

Marmottant P, Villermaux E, Claner C (2000) Transient surface tension of an expanding liquid sheet. J Colloid Interface Sci 230:29

Mourougou-Candoni N, Prunet-Foch B, Legay F, Vignes-Adler M, Wong K (1997) Influence of dynamic surface tension on the spreading of surfactant solution droplets impacting onto a lowsurface-energy solid substrate. J Colloid Interface Sci 192(1):129

Rafaï S, Bonn D, Boudaoud A (2004) Spreading of non-Newtonian fluids on hydrophilic surfaces. J Fluid Mechanics 513:77 
Svitova T, Hoffmann H, Hill RM (1996) Trisiloxane surfactants: surface interfacial tension dynamics and spreading on hydrophobic surfaces. Langmuir 12(7):1712

Van Valkenburg JW (1982) Adjuvants for Herbicides. chap. Terminology, classification, and chemistry. Weed Science Society of America, Illinois, pp. 1-9

Wirth W, Storp S, Jacobsen W (1991) Mechanisms controlling leaf retention of agricultural spray solutions. Pesticide Sci 33(4):411
Xu L, Zhang WW, Nagel SR (2005) Drop splashing on a dry smooth surface. Phys Rev Lett 94:184505

Yarin AL (2006) Drop impact dynamics: splashing, spreading, receding, bouncing. Annu Rev Fluid Mechanics 38:159

Zhang XG, Basaran OA (1997) Dynamic surface tension effects in impact of a drop with a solid surface. J Colloid Interface Sci 187(1):166 